\title{
Criminologie
}

\section{Les progrès et leurs effets pervers dans l'application de la justice des mineurs : une perspective comparative}

\section{Uberto Gatti}

Volume 26, numéro 2, 1993

La criminologie comparée. Hommage à Denis Szabo

URI : https://id.erudit.org/iderudit/017341ar

DOI : https://doi.org/10.7202/017341ar

Aller au sommaire du numéro

\section{Éditeur(s)}

Les Presses de l'Université de Montréal

ISSN

0316-0041 (imprimé)

1492-1367 (numérique)

Découvrir la revue

Citer cet article

Gatti, U. (1993). Les progrès et leurs effets pervers dans l'application de la justice des mineurs : une perspective comparative. Criminologie, 26(2), 103-120. https://doi.org/10.7202/017341ar
Résumé de l'article

One of the most important developments in juvenile justice systems in the western countries over this century has been the 1970s crisis of the well established welfare model, a crisis based on the lack of rights in juvenile procedures, the ineffectiveness of treatment interventions and the failure to decrease delinquency in society.

The reaction to this situation assumed different forms in different contexts, and showed numerous contradictions.

In some countries the justice model, an adultisation of juvenile justice, became dominant; in other countries the «back to justice " movement was not accepted, and other models developed.

In the actual organisation of juvenile justice some trends emerged : deinstitutionalisation, diversion and community alternatives to custody, the utilisation of private resources and volunteers inside a public network and bifurcation between serious and normal offenders.

The crisis of the welfare model and critical aspects of the justice model for juveniles has stimulated experts and policy makers to search for new paradigms, such as, for exemple, delinquency management, reparation and mediation, which probably represent important elements of the future of juvenile justice systems. 


\section{LES PROGRÈS ET LEURS EFFETS PERVERS DANS L'APPLICATION DE LA JUSTICE DES MINEURS : UNE PERSPECTIVE COMPARATIVE ${ }^{1}$ \\ Uberto Gatti ${ }^{2}$}

One of the most important developments in juvenile justice systems in the western countries over this century has been the 1970s crisis of the well established welfare model, a crisis based on the lack of rights in juvenile procedures, the ineffectiveness of treatment interventions and the failure to decrease delinquency in society.

The reaction to this situation assumed different forms in different contexts, and showed numerous contradictions.

In some countries the justice model, an adultisation of juvenile justice, became dominant; in other countries the "back to justice movement was not accepted, and other models developed.

In the actual organisation of juvenile justice some trends emerged : deinstitutionalisation, diversion and community alternatives to custody, the utilisation of private resources and volunteers inside a public network and bifurcation between serious and normal offenders.

The crisis of the welfare model and critical aspects of the justice model for juveniles has stimulated experts and policy makers to search for new paradigms, such as, for exemple, delinquency management, reparation and mediation, which probably represent important elements of the future of juvenile justice systems.

\section{INTRODUCTION}

La justice des mineurs est un champ de la recherche criminologique des plus explorés et ce dans de nombreux pays. L'ensemble des connaissances qui se sont accumulées avec le temps fournissent donc nombre de données intéressantes et utiles.

Ainsi réalisée dans différents contextes, la recherche procure des pistes de réflexion importantes et la comparaison, à un niveau international, semble désormais nécessaire afin d'évaluer l'impact des institutions qui interviennent dans la question de la déviance des jeunes. Nous ne pouvons ici qu'être d'accord avec Szabo (1986) lorsqu'il affirme que «la dimension

1. Traduction française révisée et mise au point par Andrée B. Fagnan, agente de recherche au Centre international de criminologie comparée.

2. L'auteur est professeur à l'Istituto di Criminologia e Psichiatria Forense, Università degli Studi di Genova, Via De Toni 12, 16132 Genova, Italia. 
comparative devient de plus en plus une fonction usuelle, voire indispensable, de l'explication des phénomènes aussi bien que de l'action politique».

Les systèmes de justice des mineurs qui s'étaient développés dans différents pays occidentaux, dès la fin du XIX ${ }^{\mathrm{e}}$ siècle, et selon des principes et des perspectives similaires, sont longtemps restés fondamentalement inchangés. C'est au cours des années 1960 qu'ils furent confrontés à une critique de plus en plus vive, ce qui donna lieu à une série d'innovations et de transformations qui ont caractérisé surtout les deux dernières décennies.

Le modèle qui a servi de point de départ, dans de nombreux pays, aux législations s'adressant aux mineurs est le modèle américain de la Cook Country Juvenile Court, créé à Chicago, en 1899.

Les principes sur lesquels reposait la création d'un Tribunal pour enfants, distinct de celui des adultes, étaient issus d'un mouvement réformateur américain de l'époque. On entendait contrer, par une intervention publique, les nombreux problèmes sociaux dus à l'industrialisation et aux vagues migratoires. (Rappelons qu'à Chicago, première ville à accueillir un Tribunal pour enfants, la population tripla entre 1880 et 1890 , en raison surtout d'une forte immigration.) On souhaitait aussi utiliser systématiquement de nouveaux moyens, empruntés aux sciences humaines, afin d'améliorer le développement psycho-social des enfants.

Selon la doctrine du parens patriae, ce Tribunal avait le droit de rompre les liens d'un mineur avec sa famille d'origine, voire de se substituer à cette dernière, dans toute situation où le bien-être du jeune était menacé. C'était dans l'intérêt du mineur qu'on maintenait des procédures informelles, jugeant que le délit commis ou le comportement déviant étaient moins importants que les besoins spécifiques du jeune pour qui on s'efforçait de choisir un traitement approprié. Un mineur qui présentait des carences sociales et familiales était alors considéré comme un sujet à soigner plutôt qu'un délinquant à punir. L'intervention visait à comprendre les causes de l'inadaptation afin d'aider, d'éduquer et de socialiser le jeunc, plus qu'à établir sa responsabilité, de façon à lui infliger une peine juste. Dans cette optique, les droits formels et la défense légale jouaient un rôle marginal.

Cette manière de voir fit en sorte que l'intervention judiciaire s'étendit à une large proportion de jeunes, formée non seulement de délinquants au sens de la loi mais aussi de mineurs déviants, marginaux ou présentant des carences familiales et sociales.

Selon les lignes directrices du modèle de la réducation, un mineur pouvait être enlevé à sa famille lorsqu'il se trouvait dans une situation de danger potentiel. Dans l'évaluation de ce danger et dans un souci d'éduca- 
tion, la durée de la prise en charge par le Tribunal n'était pas nécessairement fixée à l'avance, mais réévaluée au fur et à mesure de l'évolution du cas.

Un vaste réseau d'institutions de rééducation des mineurs fut alors créé et un imposant appareil de services sociaux fut progressivement mis sur pied afin d'appliquer les décisions du Tribunal pour enfants et de poursuivre les objectifs propres à leur rééducation.

Cette nouvelle approche inspira les législations de différents états américains et de plusieurs pays européens. Ainsi, dans la première moitié du $\mathrm{XX}^{\mathfrak{e}}$ siècle, on vit apparaître nombre de mesures légales spécialement conçues pour les mineurs déviants : le Children Act de 1908 crée le Tribunal pour enfants en Angleterre; le Jugendwohlfahrtsgesetz de 1922 et le Jugengerichtsgesets de 1923 sont deux textes législatifs majeurs sur lesquels se fondent la réducation des mineurs en Allemagne; la loi du 22 juillet 1912 établit les Tribunaux pour enfants en France; et le R.D.L. n. 1404 de 1934 institue le Tribunal pour enfants en Italie.

\section{DU MODÈLE «BIEN ÊTRE SOCIAL »AU MODĖLE «JUSTICE»}

Mises à part quelques critiques isolées et marginales, les législations pour mineurs jouirent, pendant une longue période, d'un large consensus. Et c'est justement aux États-Unis, pays qui avait amorcé le développement du mouvement des Cours juvéniles, qu'apparurent, dans les années 1960. les premières attaques systématiques contre l'idéologie de ces institutions.

Ces critiques donnèrent lieu à de nombreux changements qui furent apportés, dans les années 1960 et 1970, par une série de décisions de la Cour suprême des États-Unis. Ces décisions accordaient plusieurs droits aux mineurs aux prises avec le système de justice : le droit de recourir à un avocat de la défense, le droit à la notification des imputations, le droit d'en appeler, etc. Selon cette nouvelle orientation, c'est-à-dire celle des garanties légales, il n'était plus possible de condamner un mineur sans que tous ses droits soient respectés.

Les attaques contre le modele de la rééducation sont aussi venues d'autres fronts :

- une série d'analyses critiques, de type historique, criminologique et sociologique mirent en relief l'ambiguïté d'un système qui voulait concilier artificiellement contrôle et aide, punition et thérapie, obligeait des mineurs a «guérir», même si ce devrait être contre leur gré. élargissant, du coup, le contrôle social s'exercé sur ces jeunes (Platt, 1977); 
- certaines enquêtes démontrèrent que, à délits égaux, les mineurs pouvaient être, au nom de leur protection, plus sévèrement punis que les adultes (Dünkel, 1990);

- des recherches nombreuses et systématiques mirent à jour l'inefficacité du traitement quant à la réinsertion sociale des jeunes et la réduction du taux de récidive (Martinson, 1974);

- certaines études critiquèrent l'intervention des experts travaillant au sein du système judiciaire pour mineurs, en mettant en évidence comment leurs pratiques étaient influencées par le sens commun, par l'organisation des structures et de la routine qu'elles entraînent plutôt que par des connaissances professionnelles spécifiques (Pratt, 1989);

- la croissance de l'inquiétude sociale quant au problème de la criminalité appelait des politiques basées sur la loi et l'ordre et une justice plus sévère :

- une certaine tendance culturelle allait dans le sens d'une plus grande émancipation des jeunes dans tous les domaines, et donc de leur plus grande responsabilité dans le domaine pénal, et allait jusqu'à exiger des jeunes délinquants qu'ils répondent de leurs actes comme le résultat d'un choix plutôt que la conséquence de carences familiales ou autres (De Leo, 1990);

- l'évolution dans le champ des théories sur les causes de la délinquance alimentait aussi la critique de l'approche de la réhabilitation : d'abord, les théories interactionnistes mettaient en relief le potentiel criminogène des institutions destinées au contrôle et au traitement de la délinquance et, plus récemment, le développement des théories criminologiques axées sur l'importance de la situation facilitant le crime, détournait l'intérêt des explications voulant que des problèmes individuels ou sociaux puissent faire évoluer la personnalité des mineurs vers la délinquance (Tutt, 1991).

Dans le domaine du droit des mineurs, on constata donc, une inversion importante des tendances. On allait s'éloigner du modèle de la réhabilitation, et se rapprocher d'un modèle rappelant le droit des adultes. Ce mouvement parti des États-Unis et du Canada et qui s'étendit jusqu'à certains pays européens, porté par des motivations diverses et des idéologies divergentes, ne se concrétisa pas en un modèle clair, cohérent et linéraire, mais se développa de façon plutôt incertaine, non concertée et parfois contradictoire. Aux États-Unis, par exemple, le recours à un avocat de la défense, même s'il était inscrit dans la loi, ne fut pas respecté dans une large mesure. En effet, plusieurs recherches empiriques ont démontré que moins de la moitié des mineurs jugés ont été assistés par d'un avocat (Clarke et Kock, 1980; Aday, 1986 : Bortner, 1982 ; Feld, 1988). Paradoxalement, ces études 
ont aussi montré que la présence d'un avocat de la défense n'était pas nécessairement dans l'intérêt des mineurs. Dans une recherche récente et très intéressante, portant sur six états américains, Feld (1988) a découvert que la présence d'un avocat de la défense pouvait même entraîner une plus grande sévérité de la sentence.

Soulignons aussi qu'en Ontario et dans d'autres régions du Canada un système davantage fondé sur les garanties légales semble avoir produit une augmentation du nombre des mineurs gardés dans des institutions fermées (Hackler, 1987). De la même façon, aux États-Unis, un nombre croissant de mineurs sont emprisonnés sur de plus longues périodes (Bortner, 1988).

La nouvelle orientation du droit des mineurs, qui s'inspire davantage de leurs droits et leurs responsabilités que du modèle thérapeutique, a eu une certaine répercussion dans le monde entier, mais son application a varié selon les pays. La difficulté d'application du modèle «justice» dans le traitement socio-pénal des mineurs - modèle où l'individualisation aurait dû être possible afin de contrer les éléments d'injustice potentielle et qui aurait dû atténuer les injustices causées par le modèle «bien être social» - a incité les autorités et les agents du droit à rechercher de nouvelles solutions. Par exemple, certains pays européens n'ont retenu que quelques aspects du nouveau modèle. Les transformations réelles du droit des mineurs ne sont donc pas faciles à cerner et leurs applications semblent plutôt fort complexes, mais il demeure que certaines tendances de fond s'y sont profilees.

\section{RÉDUCTION DE L'INTERVENTION JUDICIAIRE}

Il est un élément commun à toutes les politiques criminelles actuelles dans le domaine du droit des mineurs, c'est celui de réduire le plus possible l'intervention judiciaire, en la limitant à des cas et à des situations bien définies.

Au cours des années 1960 , on élabora quelques hypothèses d'intervention destinées à maintenir les jeunes auteurs de délits en dehors du système de justice. Ces hypothèses reposaient sur la conviction que les effets de ce système ne répondaient absolument pas aux attentes du corps social. En effet, stigmatisation, renforcement de l'identité criminelle, progression dans la déviance, marginalisation sociale et forts taux de récidive se révéleront aux yeux de nombreux experts comme des effets inhérents au système pénal, système fortement bureaucratisé, détaché de la communauté et difficile à orienter vers des objectifs rationnels.

Que certaines législations aient abandonné le concept de «délit statutaire» (actes interdits aux mineurs, mais permis aux adultes), traduit remarquablement le courant qui cherche à réduire l'intervention judiciaire, à laquelle il ne s'agit plus de recourir pour contrôler des comportements déviants génériques, mais à laquelle, on ne peut faire appel que dans le cas de délits, au sens de la loi, qui s'appliquent à tous. 
Un autre aspect de la réduction de l'intervention judiciaire réside dans la tendance récente à augmenter l'âge de la responsabilité pénale. Comme le précise Dünkel (1991), l'âge de la responsabilité pénale a été élevé de 14 à 18 ans en Roumanie (1977), de 9 à 13 ans en Israël (1977), de 12 à 16 ans à Cuba (1979), de 7 à 12 ans au Canada, de 14 à 16 en Argentine (1983) et de 14 à 15 ans en Norvège (1987). Il est aussi intéressant de noter que, dans certains pays et dans un souci de désengorgement pénal, les jeunes adultes peuvent être jugés comme des mineurs. Faut-il rappeler toutefois que, contrairement à la tendance générale observée dans d'autres pays et même si le fait est très contesté, il est possible de transférer un jeune impliqué dans des délits graves et répétés, de la juridiction des mineurs à celle des adultes.

En général, la théorie de la déjudiciarisation, élaborée durant les années 1960 et mise en pratique dans de nombreux pays, découla d'un impératif : éloigner très tôt les mineurs du système de justice pénale, en leur offrant des alternatives, soit qu'ils évitent tout type d'intervention, soit, ce qui est plus fréquent, qu'ils bénéficient d'une intervention de type socio-éducatif. Une intervention socio-éducative peut être imposée au lieu d'une mesure pénale ou, suivant d'autres modèles, peut être négociée (acceptée ou refusée) par le jeune et ce, sans conséquence judiciaire. C'est dans ce dernier cas seulement, lorsque le mineur est réellement et techniquement libre de s'opposer à une proposition de mesure éducative et sans que son refus puisse conduire à une reprise de la procédure pénale ou à une quelconque forme de sanction, que l'on peut alors parler de "vraie déjudiciarisation » (true diversion) (Cressey et Mc Dermott. 1973).

Les recherches américaines sur les programmes de déjudiciarisation des mineurs n'ont pas fait l'unanimité : alors que certains auteurs ont noté une réduction du taux de récidive chez les sujets soumis à ces programmes (Baron et Feeney, 1976; Blomberg, 1979; Bohnstedt, 1978) et qu'ils ont vérifié l'efficacité et le faible degré de coercition de ces derniers (Binder et Geis, 1984), d'autres, au contraire, ont constaté une augmentation des pourcentages de récidive (Lincoln, 1976; Elliott, 1978), une sélection inappropriée des clients (Dunford, 1977), un prolongement excessif du contrôle, voire au-delà des limites temporelles des mesures judiciaires (Frazier et Cochran, 1986), et une expansion générale du système de contrôle au lieu de sa réduction (Polk, 1984). Cet effet paradoxal s'est toutefois peu vérifié en Europe; en Italie, par exemple, il est clair que le nombre de mineurs ayant fait l'objet de mesures judiciaires a globalement diminué ces dernières années. 


\section{DÉSINCARCÉRATION ET DÉSINSTITUTIONNALISATION}

Ces dernières années, tous les réformateurs se sont donné comme objectif de réduire le nombre des mineurs détenus ou gardés dans tout type d'institution fermée.

Les critiques sur la prison sont connues et largement partagées par la communauté scientifique. Les experts, les administrateurs, les responsables politiques de plusieurs pays et en particulier les organisations internationales se sont prononcés avec détermination sur cette question.

Ainsi, l'Assemblée générale des Nations Unies a adopté, en 1985, un «Ensemble de règles minima des Nations Unies concernant l'administration de la justice pour mineurs » (Règies de Beijing), selon lesquelles la privation de la liberté devrait être une mesure exceptionnelle, adoptée en dernier ressort et dont la durée serait réduite au minimum.

La gravité et l'importance du problème des mineurs en prison ont par la suite incité les Nations Unies à préparer un « Ensemble de règles minima pour la protection des mineurs privés de liberté», règles approuvées en 1990 et qui prévoient une série de limitations et de garanties. Les termes "privation de liberté » sont à entendre ici dans un sens élargi. En proposant une définition très englobante, on a voulu donner des indications d'ordre substantiel et éliminer le risque de remplacer les prisons par des institutions fermées, différentes dans la forme mais similaires sur le fond. Ces normes insistent encore sur le fait que l'incarcération des mineurs devrait être une mesure utilisée en dernier recours.

Une orientation encore plus décisive a été adoptée par la Recommandation n.R. (87) 20 du Conseil de l'Europe (Réactions sociales à la délinquance juvénile) qui affirme que le système pénal pour mineurs doit avoir comme objectif la suppression de l'emprisonnement.

Les indications internationales à propos de l'emprisonnement des mineurs ont été bien peu observées et, dans certains cas, ne constituent qu'une énonciation rhétorique qui cache une réalité répressive. Elles demeurent cependant un point de référence important pour plusieurs pays tels la France, l'Italie, la Belgique, l'Allemagne, le Danemark, la Norvège, la Hollande et la Suède qui ont, ces dernières années, adopté des législations et des pratiques judiciaires respectueuses des normes internationales. Ces pays ont ainsi réduit considérablement la probabilité d'envoyer des mineurs en prison et, du coup, diminué radicalement tous les types d'institutionnalisation. 


\section{LES SOLUTIONS DE RECHANGE À LA DÉTENTION}

L'utilisation de plus en plus répandue de mesures de liberté surveillée, comme solution de rechange à la prison, caractérise tous les systèmes de justice occidentaux.

Le coût excessif de la détention, la conscience des effets néfastes de la prison sur des sujets encore en formation, la volonté de rendre la communauté responsable quant au problème social de la délinquance juvénile, ont conduit à la multiplication de mesures de contrôle en milieu ouvert.

À côté des mesures traditionnelles, comme celle de la "probation », largement appliquée quand il s'agit de mineurs, de nouvelles formes d'intervention se sont développées, comme le «traitement intermédiaire» (Boutom et al., 1985), traitement intensif, mais ou le sujet demeure au sein de la communauté. Née en Angleterre, cette façon d'intervenir se situe comme l'indique son nom, dans une position intermédiaire, entre la liberté surveillée et le placement en institution. Le traitement comprend des activités d'éducation sociale, de scolarisation, de formation professionnelle et de détente, le tout dans le but de substituer un comportement «prosocial» au comportement déviant. Les activités se déroulent dans des «centres de jour» où les jeunes passent la journée ou, dans certains cas, la fin de semaine.

Dans la foulée, en Europe continentale, dans le cadre d'une vaste opération de politique sociale, on a développé différents programmes afin de remplacer l'intervention judiciaire. Ces programmes sociaux étaient décentralisés et destinés davantage à favoriser une meilleure intégration des jeunes dans la société qu'à contrer directement le problème de la criminalité.

En Italie, par exemple, de nombreuses innovations, surtout dans les villes du Nord, la partie la plus développée du pays. La loi de 1977 en fournissait l'occasion : à l'intérieur d'un vaste programme de décentralisation, elle transférait les compétences relatives à la gestion des mesures de rééducation, du Ministère de la Justice aux municipalités, créant ainsi des conditions favorables à l'innovation et à l'expérimentation (Gatti et Verde, 1988). Plusieurs municipalités ont donc organisé des programmes de prévention et de réhabilitation de type communautaire, liês à une importante opération de désinstitutionnalisation visant l'abolition des maisons de correction et favorisant plutôt l'organisation de petites structures d'éducation ouvertes (foyers de groupe). Au début, ces programmes furent accueillis avec méfiance, voire même avec hostilité, par les instances du contrôle social formel, ce qui eut comme conséquence d'augmenter les emprisonnements et les mesures pénales chez les mineurs. Une acceptation substantielle des nouvelles politiques locales succéda toutefois à cette première réaction négative et aboutit finalement à une importante réduction des mesures pénales (Bandini et Gatti, 1987). 
Dans la ville de Gênes (commune de 700,000 habitants), par exemple, où il n'existe plus de maisons de ré́ducation fermées, mais où des foyers et divers autres types de prise en charge au sein de la comunauté les ont remplacées, le nombre de mineurs en prison a remarquablement diminué moins de dix.

Le programme réalisé par la Mairie de Gênes repose sur les principes suivants : la désinstitutionnalisation; l'abolition de la division en diverses catégories d'assistance auxquelles correspondaient, avant 1977, différents services sociaux; l'abandon de la logique de la réćducation en faveur d'une perspective qui privilégie les besoins des mineurs; enfin, la territorialité. L'objectif fondamental de ce programme est de s'opposer à la stigmatisation et à la marginalisation des mineurs; aussi vise-t-il à éloigner du système judiciaire, et ce dès le moment où ils y seraient amenés, les jeunes accusés de délits ou considérés comme déviants.

\section{L'ÉLARGISSEMENT DU FILET PÉNAL}

Les solutions de rechange à la détention, d'abord accueillies favorablement, n'ont tout de même pas échappé aux critiques par la suite. En effet, des auteurs (Austin et Krisberg, 1982; Cohen, 1985) ont mis en évidence le phénomène de "l'élargissement du filet pénal » (net widening) : ce phénomène apparaît lorsqu'une mesure de rechange devient une mesure supplémentaire plutôt que de se substituer à une autre. Il se peut, en effet, qu'une mesure de rechange, par exemple, qui remplacerait la peine de prison, soit appliquée à un individu qui autrement n'aurait été l'objet d'aucune mesure pénale, ou tout au plus se serait vu soumis à une mesure encore moins répressive que la nouvelle.

Ainsi, ce qu'on avait conçu comme une solution de rechange pourrait donc se révéler un nouvel instrument de contrôle s'ajoutant à ceux déjà existants. De plus, dans les pays où les mesures de rechange sont particulièrement répandues, on a constaté, simultanément, une augmentation des incarcérations. Se référant surtout à la situation des États-Unis, de l'Angleterre et du Canada. Cohen (1985) démontre comment la mise en place de mesures de rechange a conduit à un accroissement du nombre global de personnes prises au filet du système pénal (wider net), à une extension des mesures plus sévères (denser net) et à une prolifération des agences impliquées dans le contrôle social (different nets).

Plus récemment, une phase de révision a succédé à celle des fortes critiques contre les mesures de rechange. Cette «critique de la critique» estime trop simplistes et pas suffisamment vérifiées les thèses de «l'élargissement du filet pénal». La recherche de McMahon (1990) est un exemple de cette nouvelle tendance : elle y précise que les statistiques, à partir desquelles les hypothèses d'élargissement du filet pénal ont été construites, 
n’ont pas été analysées de façon adéquate. Les chercheurs, en effet, auraient considéré le nombre absolu des détenus en prison plutôt que celui des détenus par 100000 habitants; ils auraient fait la somme arbitraire des détenus et des sujets en probation - ne tenant pas compte du fait que la probation dure habituellement plus longtemps que la peine de détention; ils n'auraient pas pris en considération certaines particularités des différents types de prison - par exemple celles des prisons fédérales; et ils n'auraient pas fait clairement la différence entre les entrées et les présences en prison. À la lumière de résultats de ses propres recherches, McMahon n'exclut pas la possibilité d'un phénomène d'élargissement du filet pénal, mais conclut qu'un tel phénomène doit encore être démontré.

La situation apparaît donc plus complexe que prévu. Ainsi, en Allemagne, on a mené certaines études empiriques dont les résultats ne confirment pas l'hypothèse d'un élargissement du réseau pénal : entre autres, l'hypothèse voulant que des sujets aient été soumis à des programmes de rechange alors qu'avant l'instauration de ces programmes ils auraient été acquittés sans faire l'objet d'aucune mesure, ne se vérifie pas - l'enquête de Heinz (1989) sur les délinquants juvéniles est significative à cet égard. Par contre, et toujours en Allemagne, Dünkel (1991) constate qu'il est loin d'être prouvé que les mesures de détention «mitigées", par exemple l'emprisonnement de fin de semaine, soient effectivement plus répressives que certaines mesures de rechange à la prison, par exemple la participation obligatoire à un cours de formation sociale d'une durée de six mois.

En tenant compte des états de recherche rappelés précédemment, on ne peut accepter, sans critique aucune, l'avènement des mesures de rechange pas plus qu'on ne peut les condamner trop vite en présumant de leur incapacité à réduire le recours à l'incarcération. Si ces mesures ne sont pas en elles-mêmes suffisantes pour y arriver, il est raisonnable de penser qu'elles concourent à faire fléchir la pratique de l'emprisonnement lorsqu'elles s'inscrivent dans une politique criminelle cohérente et qui s'attache tant à la législation qu'à l'application de là norme.

\section{LES DEUX JUSTICES}

Dans plusieurs pays occidentaux, le système de justice pour mineurs semble revêtir l'apparence de Janus, l'antique dieu romain aux deux visages (Bortner, 1988) : un visage bienveillant, tourné vers les nombreux mineurs jugés comme étant de «pas trop graves déviants» et pour lesquels on active fréquemment des processus de déjudiciarisation ou propose des mesures de rechange à la prison; et un autre visage, autoritaire, qui va de pair avec l'utilisation des outils judiciaires, prison ou autres mesures de contrôle très répressives, destiné aux mineurs jugés plus dangereux. 
Ces dernières années, on a aussi, quoique de manière peu précise, conçu des théries sur le «contrevenant grave-violent-chronique » (seriousviolent-chronic offender), catégorie de délinquants auxquels on attribue la responsabilité d'une bonne part de la délinquance et en particulier de la délinquance violente. On a cependant noté que le processus de catégorisation qui classe un mineur dans le groupe des contrevenants graves-violentschroniques ne tient pas seulement compte des délits ou des antécédents pénaux, mais aussi d'autres variables, telles une attitude d'opposition et une inaptitude présumée aux programmes d'éducation et de réhabilitation.

En outre, on a observé que le processus qui conduit à la définition de «sujet dangereux» en est un interactif, influencé par les objectifs, par les idéologies et par la structure organisationnelle de l'appareil de contrôle qui peut, selon le cas, élargir ou restreindre ses propres tentacules. On a de plus remarqué que ce processus de définition est aussi influencé par le degré d'inquiétude sociale quant aux problèmes de délinquance.

Le processus de catégorisation des jeunes dits délinquants a aussi souleve une autre question quant à l'application du droit des mineurs : la surpénalisation des jeunes appartenant aux classes sociales les plus pauvres, aux groupes marginaux et aux minorités ethniques. On a noté, entre autres, que ce sont souvent des jeunes les plus défavorisés du point de vue de leurs conditions familiales et sociales qui se trouvent le plus lourdement réprimés, à qui, par exemple on réserve surtout la prison. De même, en Italie, où la prison est de moins en moins utilisée dans le cas des mineurs, on y recourt toutefois plus volontiers dans le cas de jeunes étrangers, considérés comme plus difficilement contrôlables.

\section{8. «L'INGÉNIERIE SOCIALE » ET LA « GESTION DE LA DÉLINQUANCE »}

L'échec du modèle de la réhabilitation et le faible degré d'acceptation du modele «justice» dans le domaine du droit des mineurs ont souvent conduit à des législations où les modeles de «bien être social » et «justice» coexistent, mais suivant des modalités qui se sont maintes fois révélées ambiguès, incohérentes et inefficaces. Pour ces raisons, on a vu poindre certaines tentatives en vue de sortir de l'impasse actuelle, et qui semblent amener à de nouvelles formes de justice pour les mineurs.

Un nouveau modèle proposé par les législations récentes et dont les résultats ne sont pas encore clairement connus repose sur un système très complexe qui respecte les droits formels des mineurs en évitant le piège d'une approche purement punitive. L'articulation de ce système gravite autour de la définition précise des tâches assignées aux différentes institutions (judiciaires, d'assistance, privées, etc.), des limites à l'intérieur desquelles les juges et les autres agents (policiers, assistantes sociales, etc.) 
peuvent agir et des différentes possibilités de transfert d'une agence à une autre. Les objectifs de cette opération d' «ingénierie sociale" (social engineering) semblent cependant difficiles à atteindre.

En d'autres termes, il s'agit de législations qui tentent d'intégrer dans leur structure toutes les critiques faites jusqu'à aujourd'hui đes législations qui concernent les mineurs. Elles tentent de concilier l'inconciliable à travers des modèles formels toujours plus complexes. La Loi sur les jeunes contrevenants (1984) au Canada et certaines législations récentes sur les mineurs en Belgique se rapprochent de ces modèles.

Une autre tentative pour remédier aux insuccès du système de justice des mineurs, et qui est plus de l'ordre de la gestion que de la législation, consiste en un programme d'intervention visant l'efficacité : la "gestion de la délinquance» (delinquency management) (Pitts, 1988). Il s'agit d'une approche pragmatique ayant pour but l'actualisation de stratégies précises par des autorités surtout locales, et qui visent la déjudiciarisation et un moindre recours à la garde en institution pour les jeunes qui se retrouvent malgré tout sous tutelle judiciaire.

Par ailleurs, Pratt (1989) a qualifié de corporatiste (corporatism) un modèle dont les caractéristiques sont les suivantes : augmentation des possibilités de décision des autorités administratives, vaste éventail de mesures applicables par le Tribunal, centralisation des compétences et coordination des politiques, participation des agences non judiciaires et, enfin, vaste champ de contrôle de certains programmes. Ce modele de gestion, implanté surtout dans certaines villes anglaises, s'oppose en quelque sorte au modèle «justice», récusé par plusieurs ; il est géré localement par des spécialistes en droit des mineurs et se donne comme objectif l'application et la vérification d'une politique prétablie, plutôt que la réponse à des besoins individuels des mineurs ou la défense en tant que telle de leurs droits. La réalisation d'un tel programme tient à la coordination et à l'utilisation de toutes les agences et ressources, tant publiques que privées, qui s'occupent de quelque manière de délinquance juvénile.

Dans la perspective de ce programme, le jeune déviant n'est ni un délinquant à punir, ni un jeune à aider, mais devient plutôt un problème à résoudre, et ce le plus efficacement possible. C'est à cette fin qu'on privilégie une intervention axée sur le comportement du jeune qui devient alors l'objet d'un processus de restructuration. Une telle approche comporte toutefois le risque que soit transféré, des institutions aux communautés, un système de contrôle répressif, inhumain et injuste (Pratt, 1989). 


\section{LA JUSTICE DE RESTAURATION}

Depuis quelques années, de nouvelles conceptions de la justice dont l'importance est toujours plus grande tentent de remplacer par un nouveau paradigme les approches punitives traditionnelles ainsi que les approches thérapeutiques : il s'agit de réparer, d'une façon ou d'une autre, les dommages causés par un crime (Wright, 1991; Walgrave, 1991).

Alors que la justice traditionnelle réagissait à un mal - le délit - par un autre - la peine - de manière nullement bénéfique pour la société ou pour la victime, le nouveau modèle, au contraire, defini à l'aide du terme plutôt large de «justice restauratrice», propose de réagir de façon utile et positive : il entend remédier, au moins partiellement, aux dommages causés par le délit. Les projets les plus ambitieux et innovateurs s'engagent même dans la résolution de conflit entre le contrevenant et la victime en tentant de rétablir à nouveau l'équilibre et l'harmonie entre les deux parties.

Marquant un intérêt pour les victimes, cette conception de la justice, manifeste au point de pouvoir parler d'un nouveau paradigme, comprend toute une série d'interventions, de modalités de gestion des causes pénales et de mesures de toute, sorte, qui vont des programmes de réparation et de travail communautaire à ceux de conciliation et de médiation.

C'est en cherchant une sanction qui ne soit pas seulement punitive, mais aussi utile pour la société qu'on a instauré, en Angleterre, les «Ordonnances de service à la communautê » (Community Service Orders) par le biais du Criminal Justice Act de 1972. D'abord expérimentée dans six régions, ces ordonnances se sont étendues rapidement à tout le territoire national.

Le C.J.A. de 1982 allait permettre à une cour juvénile d'imposer une Ordonnance de service à la communauté aux jeunes inculpés à partir de l'âge de 16 ans. Cette mesure comporte l'obligation d'effectuer un travail sous forme de service à la communauté pendant un nombre d'heures déterminé par le Tribunal, qui va d'un minimum de 40 heures à un maximum de 240 heures à l'intérieur d'une peine d'un an. Cette mesure est généralement précédée d'une enquête sociale, mais, afin qu'elle puisse s'appliquer, il est nécessaire d'avoir l'accord de la personne qui y serait soumise. Si la personne à qui on impose une telle ordonnance n'effectue pas régulièrement le travail exigé, son cas peut être à nouveau examiné par le Tribunal qui peut lui permettre de compléter le travail ou lui imposer une peine différente.

A la suite de l'expérience anglaise, les Ordonnances de service à la communauté ont été introduites dans plusieurs législations, telles celles des États-Unis, de la France, du Danemark, de la Hollande, de l'Italie, de l'Allemagne, du Portugal, etc., soit comme sanction de rechange à la prison, 
soit en remplacement d'une amende pour des sujets qui sont dans l'incapacité de la payer.

Plus innovateur que les précédents, un modele qui s'inspire de la médiation entre le délinquant et la victime allait apparaître. On l'expérimente pour la première fois à Kitchener (Canada) en 1975 et il est rapidement adopté par d'autres pays. Les programmes prévus dans ce modèle peuvent être assez formels et gérés par des agents publics (police, tribunal, etc.) ou par des institutions privées et des bénévoles; ils ont pour but la réconciliation entre l'auteur d'un crime et la victime, voire un dédommagement de la victime; ils peuvent être réalisés dans un contexte de déjudiciarisation, tenir lieu de véritables sanctions ou bien de conditions à la « liberté conditionnelle».

Il est intéressant de noter qu'une recherche allemande (Dünkel, 1990) révèle que 39 pour cent des mineurs jugés par le Tribunal sont considérés aptes à une intervention de médiation, et que la médiation réussit dans 70 à 80 p.cent des cas. Des résultats similaires ont été obtenus dans une recherche menée en Autriche, où une loi récente permet aux juges de proposer aux mineurs inculpés une intervention de médiation, conduite alors par un travailleur social expert en la matière. Si l'auteur et la victime arrivent à un accord sur ce qui doit être fait, le procès est affaire classée; dans le cas contraire, le jeune délinquant verra son procès suivre son cours.

La recherche empirique a révélé qu'une minorité de victimes n'est pas disposée à participer à une procédure de médiation, alors que la majorité, au contraire, accepte volontiers cette solution et se déclare, en général, satisfaite de ce type de réaction au crime (Umbreit, 1991).

Il demeure évident, cependant, que dans certaines situations, une procédure de médiation est impossible, par exemple quand la victime est inconnue ou qu'elle s'oppose à ce genre de règlement. Mais dans un nombre important de cas, peut-être plus nombreux qu'on peut l'imaginer, la justice du type de la réparation peut constituer une troisième voie eu Égard aux modèles du traitement et de la rétribution.

\section{CONCLUSION}

L'application de la justice des mineurs semble, par une sorte de fatalité, entrâner des effets pervers aux conséquences parfois très lourdes qui prennent souvent une importance plus grande que les résultats escomptés.

Rappelons d'abord que l'approche thérapeutique qui a pour but d'aider et de traiter les jeunes souffrant de carences de toutes sortes s'est souvent avérée un instrument de répression contre laquelle ces jeunes n'avaient guère de recours légaux - il est même arrivé que le recours aux droits formels ait donné lieu à une augmentation de la peine. De même, il faut 
souligner que certains efforts de rationalisation ont eu un effet pervers particulier : la marginalisation des jeunes appartenant aux classes sociales les plus défavorisées. De plus, faut-il noter, la recherche de solutions de rechange à la prison a abouti, dans certains cas, en un élargissement du réseau de contrôle, sans réduire pour autant le recours à l'incarcération.

À l'optimisme des premiers réformateurs qui avaient crế les Tribunaux pour enfants a donc succédé un fort scepticisme qui caractérise la tendance actuelle : avant tout, chercher à éviter les dommages causés par le système de justice lui-même. Déjudiciarisation, intervention minimale, recherche de solutions de rechange, etc., voilà donc les éléments du nouveau discours.

En guise de conclusion, nous pouvons aussi affirmer que le modèle «justice» n'a pas remplacé le modèle «bien-être-social», et l'observation vaut surtout pour l'Europe continentale. Il est juste de dire, cependant, que le système de justice des mineurs tient davantage compte maintenant des droits de ces derniers, mais sans verser dans le formalisme, ou adopter l'approche rétributive de la justice des adultes.

Il nous semble, donc, que de nouvelles orientations et de nouveaux paradigmes sont en train d'émerger : une plus grande attention aux besoins des victimes de délit a suscité le développement de programmes de dédommagement et de médiation qui, même s'ils ne sont pas utiles dans tous les cas, sont certainement intéressants tant du point de vue des valeurs qui les sous-tendent que de leurs résultats; la conscience de la lourdeur et de la distorsion des appareils bureaucratiques a conduit a l'utilisation croissante de structures privées, plus flexibles que les publiques. Ajoutons quant à ce dernier point que la diversification et la multiplication d'agences privées a permis l'élaboration de modèles complexes qui unissent dans un seul réseau, souvent coordonné par des autorités locales, tous les agents qui interviennent auprès des jeunes délinquants ; ainsi, de nouveaux intervenants et experts en justice juvénile ont uni leurs efforts à ceux des magistrats et des travailleurs sociaux traditionnels.

Même si le risque des effets pervers, ceux qui particulièrement seraient issus d'une nouvelle bureaucratie, cette fois admnistrative plutôt que judiciaire, subsiste toujours, il est permis de conclure que les nombreuses initiatives prises en regard de l'application de la justice des mineurs laissent entrevoir des développements prometteurs.

Soulignons, en terminant, que, afin de comprendre tant les conséquences favorables que les effets pervers de l'application des lois et des normes visant à faire face au problème de la délinquance juvénile, la recherche empirique semble indispensable (Szabo, 1981). Même si elle ne peut atteindre à elle seule l'objectif ambitieux de fournir les lignes directrices de choix politiques, elle joue un rôle important. En effet, elle 
concourt à élargir le champ des connaissances, à mettre en évidence l'inefficacité de certains projets et à stimuler, par ailleurs, la formulation de nouvelles orientations et de programmes innovateurs.

\section{BIBLIOGRAPHIE}

ADAY, D.P. (1986). * Court Structure, Defence Attorney Use and Juvenile Court Decisions ", Sociological Quarterly, 27, p. 107.

AUSTIN, J. et B. KRISBERG (1981), «Wider, Stroger and Differents Nets : the Dialectics of Criminal Justice Reform », Journal of Research in Crime and Delinquency, janvier, p. 165.

BANDINI, T. et U. GATTI (1987), Delinquenza giovanile, Giuffrè, Milano.

BARON, R. et F. FEENEY (1976), Juvenile Diversion Trough Family Counselling, U.S. Government Printing Office, Department of Justice, Washington, D.C.

BINDER, A. et G. GEIS (1984), « Ad Populum Argumentation in Criminology : Juvenile 'Diversion as Rhetoric ", Crime and Delinquency, 30, p. 624.

BLOMBERG, T. (1979), «Diversion from the juvenile court : a review of evidence " in F. FAUST et P. BRANTINGHAM (éds.), Juvenile Justice Philosophy, West, St. Paul.

BOHNSTEDT, M. (1978), «Answers to Three Questions about Juvenile Diversion», Journal on Research on Crime and Delinquency, 15, p. 109.

BORTNER, M.A. (1982), Inside a Juvenile Court : The Tarnished Ideal of Individualized Justice, New York University Press, New York.

BORTNER, M.A. (1988), Delinquency and Justice : An Age of Crisis. Mc Graw-Hill, New York.

BOTTOMS, A.E., P. BROWN, B. McWILliaMS, W. McWILliaMS et J. PRATT (1985), The National IT Survey: Some Preliminary Findings. NIFTED Annual Conference, Sheffield.

CLARKE, S.H. G.G. KOCH (1980), « Juvenile Court : Therapy or Crime Control, and Do Lawyers Make a Difference ? , Law and Society Review, 1, p. 15.

COHEN, S. (1985), Vision of Social Control. Polity Press, Cambridge.

CRESSEY, D.R., R.A. MCDERMOTT (1973), Diversion from the Criminal Justice System, National Assessment of Juvenile Correction, University of Michigan, Ann Arbor (Mich.).

De LEO, G. (1990), La devianza minorile, La Nuova Italia Scientifica, Roma.

DUNFORD, F.W. (1977), « Police Diversion : An Illusion? ", Criminology, 15, p. 335.

DÜNKEL, F. (1990), «Médiation délinquant-victime et réparation des dommages. Nouvelle évolution du droit pénal et de la pratique judiciaire dans une comparaison internationale $*$, in F. DÜNKEL et J. ZERMATTEN (éds.), Nouvelles Tendances dans le Droit Pénal des Mineurs. Max-Planck-Inst. für Ausland. u. Internat. Strafrecht, Freiburg i. B. 
DÜNKEL, F. (1991), «Legal differences in Europe relevant to Juvenile Criminology *. Paper presented at the 8 th International Workshop for Juvenile Criminology. Leeds (U.K.), 2-4 juillet 1991.

ELLIOTT, D.S. (1978), Diversion : A Study of Alternative Processing Practices. Behavioral Research Institute, Boulder, Col.

FELD, B.C. (1988), «In re Gault Revisited : A Cross-State Comparison of the Right to Counsel in Juvenile Court ", Crime and Delinquency, 34, p. 393.

FRAZIER, C.F., J.K. COCHRAN (1986), * Official Intervention, Diversion from the Juvenile Justice System, and Dynamics of Human Services Work : Effects of a Reform Goal Based on Labeling Theory », Crime and Delinquency, 32, p. 157.

GATTI, U., A. VERDE (1988), * S'éloigner du système pénal : une approche du problème de la délinquance juvénile en Italie $n$, Revue Internationale de Criminologie et de Police Technique, 41, p. 49.

HACKLER, J. (1987), « Increasing Confrontation in Juvenile Justice : Constrasting Changes in North America with Trends in Europe », Sixth International Workshop on Juvenile Criminology, vol. II, Acco, Leuven.

HEINZ, W. (1989), «Jugendstrafrechtsreform durch die Praxis - eine Bestandsaufnahme $*$. In Bundesministerium der Justiz (દd.), Jugendstrafrechtsreform durch die Praxis, Bonn.

LINCOLN, S.B. (1976), * Juvenile Referral and Recidivism», in R.M. CARTER et M.W. KLEIN (Éds.), Back on the Street: The Diversion of Juvenile Offenders. Prentice-Hall, Englewood Hill (N.J.).

MARTINSON, A. (1974), * What works? Questions and Answers about Prison Reform », The Public Interest, 1974, 35, p. 22.

McMAHON, M. (1990), * Net-widening : vagaries in the use of a concept », British Journal of Criminology, 30, p. 121.

PITTS, J. (1988), The Politics of Juvenile Crime. Sage, London.

PLATT, A. (1977), The Child Savers : The Invention of Delinquency. University of Chicago Press, Chicago.

POLK, K. (1984), * Juvenile Diversion : A Look at the Record », Crime and Delinquen$c y, 30$, p. 648.

PRATT, J. (1989), «Corporatism : the third model of juvenile justice », British Journal of Criminology, 29, p. 236.

SZABO, D. (1981), «L'évaluation des politiques criminelles: quelques réflections préliminaires », Revue de science criminelle et de droit pénal comparé, 1, p. 1.

SZABO, D. (1986), Science et crime. Vrin, Paris/Montréal.

TUTT, N. (1991), * The Future of the Juvenile Justice System, in J. JUNGER-TAS, L. BOENDERMARKER et P.H. VAN DER LAAN (éds.), The Future of the Juvenile Justice System. Acco, Leuven.

UMBREIT, M. (1991), * Mediating Victim Offender Conflict: from Single-site to Multi-site Analysis in the USA ", in H. OTTO et H. MESSMER (Éds.), Conflict, Crime and Reconciliation. The Organization of Welfare Intervention in the Field of Restorative Justice. Nato Advanced Research Workshop, Il Ciocco (Italy). 
WALGRAVE, L. (1991), «Mediation and Restitution as Model of a Restorative Approach: Why Would It Be Better? Expliciting the Objectives as Criteria for Research », in H. OTTO et H. MESSMER (éds.), Conflict, Crime and Reconcilia. tion. The Organization of Welfare Intervention in the Field of Restorative Justice. Nato Advanced Research Workshop, II Ciocco (Italy).

WRIGHT, M. (1991), Justice for Victims and Offenders : a Restorative Approach to Crime. M. Keynes, Open University Press. 